\title{
A Proportional-Switch Adjustment Model towards Mixed Equilibrium with Multiroute Choice Behaviour Criterion
}

\author{
Zhongxiang Huang $\left(\mathbb{D}\right.$, Xiangjun Jiang ${ }^{\mathbb{D}}$, and Wei Hao \\ School of Traffic \& Transportation Engineering, Changsha University of Science \& Technology, Changsha 410076, Hunan, China \\ Correspondence should be addressed to Xiangjun Jiang; 18874033439@163.com
}

Received 17 December 2019; Revised 5 February 2020; Accepted 6 July 2020; Published 7 August 2020

Academic Editor: Young-Ji Byon

Copyright (C) 2020 Zhongxiang Huang et al. This is an open access article distributed under the Creative Commons Attribution License, which permits unrestricted use, distribution, and reproduction in any medium, provided the original work is properly cited.

\begin{abstract}
Based on the price-quantity adjustment behaviour principle of the non-Walrasian equilibrium theory, this paper adopted a new QUE (quantity adjustment user equilibrium) criterion to formulate the route comfort choice behaviour. The purpose of the present paper is to establish a proportional-switch adjustment model which aims to reflect the route adjustment behaviour interaction between the traditional UE (user equilibrium) travellers and the QUE travellers and converge to a mixed equilibrium state. It is assumed that a group of road network travellers follow the UE criteria by choosing the travel route with the purpose of minimizing their route travel time (travel cost). In addition, the other group of travellers follow the QUE criteria by selecting the route with the largest residual capacity to achieve a more comfortable travel experience. The travel route adjustment behaviour of the two group travellers generates the dynamic traffic flow evolution towards the mixed equilibrium, and the route adjusting flow is proportional to the difference of traveller decision-making variable among the alternative routes. Simple illustrative examples are used to evaluate the performance of the proposed model, and the uniqueness and stability of the solution are demonstrated by applying the variational inequality and Lyapunov stability theorem.
\end{abstract}

\section{Introduction}

DTA (dynamic traffic assignment) model has been a focus research area for a long time with a growing influence on the traffic control and guidance promoted by the development of ITS (intelligence transportation system) emerging, leading to a greater interest in understanding the travel choice decision behaviour and the adjustment behavioural mechanisms [1-4]. Route choice behaviour is the basis of the traffic flow, and researchers have carried out a number of studies for dynamic traffic flow evolution problems to deepen the understanding of travellers' route choice behaviour decision-making mechanism [5-8]. The dynamic models are classified into five categories in the early researches: the simplex gravity flow dynamics, proportionalswitch adjustment process, network tatonnement process, projected dynamical system, and evolutionary traffic flow dynamics. The difference between the five types of methods lies in the convergence speed of the established evolutionary model and the stability in equilibrium state [9-12]. Guo et al. [13] established a link-based discrete dynamic system model and depicted the rational adjustment process of network traffic flow from disequilibrium to an equilibrium state, which formulated more general behaviour compared with the five categories of dynamical system models.

Traditional dynamic traffic assignment problem assumes that travellers' route adjustment behaviour is aimed to reduce their travel cost; therefore, dynamic traffic flow evolution models converged to the equilibrium solution with minimizing the travel cost. There are three well-known network equilibrium states in the network traffic flow analysed studies. In detail, both of them capture travellers' cost-minimization behaviour with a difference in the form of the travel cost. Furthermore, travellers choose the actual shortest route in the route decision process to form a UE (user equilibrium), and travellers shift to the route to decrease the system aggregate travel time to form an SO (system optimum). At last, travellers adjust their routes 
according to the perceived route travel time to form an SUE (stochastic user equilibrium). Although these classical traffic flow equilibrium conditions differ in the form of travel time, they share the same route rapidity choice criterion, which can embody the travellers' preference of route rapidity and reflect the price signal affection. It is apparent that all existing models in the literature were formulated based on a single route rapidity choice preference, where the route travel time is the core indicator. This approach has been widely applied in various network traffic flow models [14-18]. However, with the rapid economic growth and social development, people-oriented considerations, such as traffic safety and travel satisfaction, have been the dominant research direction [19-22].

Basically, the existing dynamic traffic flow evolution models are mainly classified according to the modelling approaches as daily and intraday, deterministic and stochastic, continuous time and discrete time, and route-based and link-based. Huang and Lam [23] established a routebased day-to-day dynamic system to depict the traffic flow adjustment process considering the network queuing, studied travellers' equilibrium path and travel time decisionmaking behaviour, and demonstrated the existence of discrete time dynamic UE solution with the iterative stability of the proposed algorithm. Secondly, Peeta and Yang [24] established route guidance control strategies and analysed the stability of dynamic traffic assignment with the example of UE objective model and SO objective model, which develops a general procedure for the stability analysis of dynamic traffic assignment problems. In addition, He et al. [25] showed that the route-based traffic flow evolution model had the path-overlapping problem and the route-flow-nonuniqueness problem caused by different initial route flow patterns. A link-based dynamic traffic flow assignment model is established, which has the classic UE solution, to depict the traveller's daily route adjustment behaviour and the evolution process of traffic flow. In order to depict the joint evolution trajectory of travellers' departure time and mode choices, Liu et al. [26] established a traffic flow dynamic model considering the impact of traveller inertia in a bimodal transportation system and analysed the dynamic interactions between transport users and traffic information provider.

With the purpose to deepen the understanding of travellers' route choice decision behaviour mechanisms, Watling and Hazelton [27] established a mathematical model to simulate the day-to-day dynamic adjustment system through the deterministic process and stochastic process models for both continuous and discrete time and described the route selection behaviour made by network travellers based on their travel experiences. Interestingly, Ehrgott et al. [28] considered several alternative principles of traveller's route decision-making behaviour and established an SUE model based on random utility theory to study this multiobjective problem, which extends the conventional SUE model with single route choice quality. In addition, Long et al. [29] proposed an SO-DTA (system optimum dynamic traffic assignment) model that accommodated the environmental objectives, which are more accurate than existing models and can capture the trade-off between emissions and travel time. Meanwhile, Hoang et al. [30] proposed a novel mathematical programming framework for the information-based SUE-DTA (stochastic user equilibrium dynamic traffic assignment) problem, which can underpin the linkage between UE and SO solutions by the FIFO (first in first out) principle. An ISM (incremental solution methods) is developed with the improvement of finding global optimal information-based SUE solution in terms of both accuracy and computation.

With the continuous improvement of science and technology, road network travellers can obtain the network information reflecting the travel environment and conditions through various methods, which are the real basis of travellers' different travel route decision-making criteria $[31,32]$. In the reality, travellers make their route decision based on their preference, such as travel safety degree, travel service degree, travel outlay, or travel time, which have been the hot issue for the travellers' behaviour research [33-40]. Traffic flows based on these route choice preferences evolve into different equilibrium trajectories and converged to different equilibrium states [41, 42]. Therefore, all kinds of traffic flows formed by travellers exist simultaneously under different criteria.

Aiming to find a link or route flow patterns in a traffic network with various route choice behaviour criteria and to describe the interaction evolution process of mixed traffic flow, extensive studies have focused on the mixed traffic flow of multiple classes of travellers in recent years. Huang and $\mathrm{Li}$ [43] grouped the travellers according to their own value of time (VOT) and further divided them into two categories with different travel disutility perception variations according to whether the ATIS device is equipped. A multiclass, multicriteria (cost versus time) logit-based traffic equilibrium assignment problem is established, which was solved by combining logit assignment with a successive average method, providing an insight into the different responses of heterogeneous travellers to the information service. Furthermore, Zhang et al. [44] considered travellers' perceived utility differences by combining the travel time disutility and the time-irrelevant travel disutility and assumed that travellers follow either UE or CN (Cournot-Nash equilibrium) routing criteria. And a multiclass multicriteria mixed equilibrium model is proposed to study this evolution behaviour. Meanwhile, a uniform road toll strategy is developed by utilising the dual theory to promote the UE-CN mixed equilibrium to the SO. Moreover, Han et al. [45] established a daily dynamic adjustment system of the traffic flow under ATIS (advanced travel information system) and analysed the influence of ATIS on the stability of the traffic network. They concluded that the route flows associated with travellers equipped with ATIS satisfied the UE conditions and the travellers without ATIS will choose their routes in accordance with the logit-based route choice probability.

To flexibly reveal the various traffic flow dynamic adjusting processes induced by different criteria of route choice behaviour, several dynamic traffic flow models have been studied recently. Zhou et al. [46, 47] established a 
discrete dynamic evolution model of traffic flow mixed equilibrium, which described traveller's multiple route adjustment behaviour with and without ATIS and simulated the evolution trajectory of traffic flow converging to UE-SUE mixed equilibrium state under given ATIS market penetration. Their research revealed the effect of the adoption of ATIS on the day-to-day flow dynamics. Bagloee et al. [48] divided travellers into two categories: perfect cooperation and perfect competition, where travellers with perfect cooperation followed the SO criterion and travellers with perfect competition were subject to the UE criterion. A UESO mixed equilibrium model is formulated by applying the nonlinear complementarity problem, which can accommodate a more general type of functions, namely, asymmetric delay functions. Moreover, Delle Site [49] separated travellers into three categories: (a) travellers equipped with predictive ATIS, (b) travellers equipped with static ATIS and subject to it, and (c) travellers not equipped with ATIS or not subject to it. They proposed an MUE (mixed user equilibrium) model to form the dynamic day-to-day processes and investigated the impacts of the inertia and the availability of ATIS with differentiated functionalities (predictive and static) on equilibrium travel times. Furthermore, the dynamic evolution models can be categorised by travellers' route choice behaviour criterion and the corresponding stable equilibrium state. Prominent examples include the single equilibrium models and the mixed equilibrium models, in which travellers follow the UE, SO, SUE, CN, or their hybrid behaviour criterion, respectively. We summarize the typical day-to-day flow dynamic models in Table 1.

Previous studies assumed that the equilibrium state of the road network is generated by the decision-making process of traveller's route rapid choice behaviour criterion, which means that travellers always shift to a less costly alternative route if such a route is available. In the dynamic evolution model of mixed equilibrium traffic flow that does not consider the route comfort choice behaviour criterion, all travellers' route adjustment behaviours conform to the cost-minimization principle, and the corresponding mixed equilibrium state is generally manifested as UE-SUE, UE-SO, UE-CN-SO, etc. This kind of mixed equilibrium only differs in the expression form of travel time, and in fact, it still takes route travel time as the travellers' only decision variable, which is inconsistent with the real transportation system. In actuality, travellers have independent characteristics, and their route choice decision behaviour is influenced by many internal factors as well as external conditions. Even under the same traffic condition and provided with the same road network information, some travellers may choose the fastest route, while others pay more attention to the overall travel comfort, such as road scenery, service level, and safety degree. In order to effectively describe the evolution process of traffic flow in road network under multiple traveller classes and multiple equilibrium criteria more effectively, Huang et al. [50] established a daily traffic flow dynamic regulation model based on the economic pricequantity regulation principle utilising the network tatonnement process method and proposed a new route residual capacity index to reflect road network travellers' comfort pursuit behaviour.

Unlike the existing studies with a single route rapidity adjustment behaviour criterion in which all the travellers were assumed to follow the same route cost-minimum behaviour mechanism, route comfort adjustment principles are considered in this study. We apply the route rapidity and comfort adjustment criteria stimulatingly to individual travel route selection behaviour, which means that individuals will comprehensively consider the route cost and the route surplus capacity to make a route decision. When applied to the traveller group, the aggregated effect is performed the way that some travellers choose the shortest route and some travellers choose the most comfortable route, as discussed in this study [51]. Specifically, travellers with route rapidity requirement are likely to choose the shortest route with the minimum route cost under the current traffic condition for their trips in the next day, and their traffic flow evolution process converges to the traditional UE state, while travellers with route comfort requirement are supposed to follow the route comfort behaviour criterion based on the current traffic condition, and their traffic flow evolves to a stable state, which is defined as QUE (quantity adjusting user equilibrium). Taking the route cost and the route surplus capacity as the decision variables for two group travellers, respectively, this paper established a dynamic proportional-switch adjustment model to depict the day-to-day evolution process and visualise the UE-QUE mixed equilibrium state formed by the interaction between the mixed traffic flows. In addition, the properties of the proposed dynamic switch model and its mixed equilibrium solution are analysed and discussed. Reasonable interpretations of these assumptions are presented to demonstrate that the route adjustment principle and the dynamic model proposed in this study have rich behavioural implications other than being a mathematical expression.

The proposed mixed traffic flow proportion-switch adjustment model with multiroute choice behaviour criterion not only reflected the heterogeneity of travellers within the different route adjustment principles but also described the different travel route adjusting process of travellers related to their different decision variables. Meanwhile, it also considered the influence of the road network traffic flow and travel costs generated by the two groups of travellers on each other's route decisions and effectively depicted the interaction relationship between the road network travellers. The research results of this paper can not only enrich the existing research on mixed equilibrium evolution by reflecting the adjustment mechanism of road network traffic flow and its equilibrium state more comprehensively but also describe the evolution process of traffic flow in line with the actual road network conditions more effectively. Hence, it improves the perception of the automatic adjustment mechanism and the changing pattern of road network traffic flow after the disturbance, which lays a foundation for the rational formulation of urban traffic congestion evacuation policy and the acquisition of reliable traffic flow prediction results. In 
TABLE 1: Typical day-to-day flow dynamic models.

\begin{tabular}{|c|c|c|c|}
\hline Authors & $\begin{array}{l}\text { Behaviour } \\
\text { criterion }\end{array}$ & Stable state & Remarks \\
\hline Guo et al. [13] & UE & $\begin{array}{l}\text { Single } \\
\text { equilibrium }\end{array}$ & $\begin{array}{l}\text { A link-based discrete dynamic system model was proposed to depict the rational } \\
\text { adjustment process of traffic flow to an equilibrium state }\end{array}$ \\
\hline $\begin{array}{l}\text { Huang and Lam } \\
{[23]}\end{array}$ & $\mathrm{UE}$ & $\begin{array}{l}\text { Single } \\
\text { equilibrium }\end{array}$ & $\begin{array}{l}\text { A route-based day-to-day dynamic system considering the network queuing was } \\
\text { formulated to study travellers' equilibrium path and travel time decision-making } \\
\text { behaviour }\end{array}$ \\
\hline $\begin{array}{l}\text { Peeta and Yang } \\
{[24]}\end{array}$ & $\mathrm{UE} / \mathrm{SO}$ & $\begin{array}{l}\text { Single } \\
\text { equilibrium }\end{array}$ & $\begin{array}{l}\text { Route guidance control strategies and a general procedure for the stability analysis } \\
\text { of dynamic traffic assignment problems were developed }\end{array}$ \\
\hline He et al. [25] & $\mathrm{UE}$ & $\begin{array}{l}\text { Single } \\
\text { equilibrium }\end{array}$ & $\begin{array}{c}\text { A link-based dynamic traffic flow assignment model was established to depict the } \\
\text { traveller's daily route adjustment behaviour and the evolution process of traffic } \\
\text { flow }\end{array}$ \\
\hline Liu et al. [26] & UE & $\begin{array}{l}\text { Single } \\
\text { equilibrium }\end{array}$ & $\begin{array}{c}\text { A traffic flow dynamic model considering the impact of traveller inertia in a } \\
\text { bimodal transportation system was investigated }\end{array}$ \\
\hline $\begin{array}{l}\text { Watling and } \\
\text { Hazelton [27] }\end{array}$ & SUE & $\begin{array}{l}\text { Single } \\
\text { equilibrium }\end{array}$ & $\begin{array}{c}\text { A mathematical model to simulate the day-to-day dynamic adjustment system and } \\
\text { the route selection behaviour made by network travellers based on their travel } \\
\text { experiences was described }\end{array}$ \\
\hline Ehrgott et al. [28] & SUE & $\begin{array}{c}\text { Single } \\
\text { equilibrium }\end{array}$ & $\begin{array}{c}\text { An SUE model based on random utility theory was performed to study the } \\
\text { multiobjective problem }\end{array}$ \\
\hline Long et al. [29] & $\mathrm{SO}$ & $\begin{array}{l}\text { Single } \\
\text { equilibrium }\end{array}$ & $\begin{array}{l}\text { A system optimum dynamic traffic assignment model that accommodated the } \\
\text { environment objectives was introduced }\end{array}$ \\
\hline Hoang et al. [30] & SUE & $\begin{array}{l}\text { Single } \\
\text { equilibrium }\end{array}$ & $\begin{array}{c}\text { A novel mathematical programming framework was investigated for the } \\
\text { information-based stochastic user equilibrium dynamic traffic assignment } \\
\text { problem }\end{array}$ \\
\hline Huang and Li [43] & SUE-SUE & $\begin{array}{l}\text { Mixed } \\
\text { equilibrium }\end{array}$ & $\begin{array}{c}\text { A multiclass, multicriteria (cost versus time) logit-based traffic equilibrium } \\
\text { assignment problem was analysed, which grouped travellers according to their } \\
\text { own value of time and further divided travellers with different travel disutility } \\
\text { perception variations }\end{array}$ \\
\hline Zhang et al. [44] & UE-CN-SO & $\begin{array}{l}\text { Mixed } \\
\text { equilibrium }\end{array}$ & $\begin{array}{l}\text { A multiclass multicriteria mixed equilibrium model that considered travellers' } \\
\text { perceived utility differences was proposed to study the evolution behaviour, and a } \\
\text { uniform road toll strategy by utilising the dual theory was developed }\end{array}$ \\
\hline Han et al. [45] & UE-SUE & $\begin{array}{c}\text { Mixed } \\
\text { equilibrium }\end{array}$ & $\begin{array}{c}\text { A daily traffic flow dynamic adjustment system was presented under ATIS } \\
\text { environment }\end{array}$ \\
\hline Zhou et al. [46] & UE-SUE & $\begin{array}{l}\text { Mixed } \\
\text { equilibrium }\end{array}$ & $\begin{array}{l}\text { A discrete dynamic evolution model of traffic flow mixed equilibrium was shown } \\
\text { to describe traveller's multiple route adjustment behaviour with and without ATIS }\end{array}$ \\
\hline Delle Site [49] & SUE-SUE-SUE & $\begin{array}{l}\text { Mixed } \\
\text { equilibrium }\end{array}$ & $\begin{array}{l}\text { A mixed user equilibrium model was proposed to form the dynamic day-to-day } \\
\text { processes and investigate the impacts of the inertia and the availability of ATIS } \\
\text { with differentiated functionalities (predictive and static) on equilibrium travel } \\
\text { times }\end{array}$ \\
\hline
\end{tabular}

Note: UE: user equilibrium; SO: system optimum; SUE: stochastic user equilibrium; CN: Cournot-Nash equilibrium.

addition, this study expands the decision influencing factors of travellers' route choice, which reflects travellers' individual characteristics more flexible, and significantly deepens the understanding of network traffic flow and improves the level of urban traffic management.

\section{Mixed Equilibrium State}

2.1. Quantity Adjustment User Equilibrium. Existing studies on the equilibrium state of traffic flow assumed that the network travellers behave in a way to minimize their travel time, which is defined as the route rapidity choice behaviour criterion in this paper. According to the different manifestation of travel time, they can be divided into three categories: the traffic flow generated by the route decisions based on actual travel time decision convergences to the traditional UE (user equilibrium) state, the traffic flow formed by the route decision according to perceived path travel time leads to an SUE (stochastic user equilibrium) state, and the traffic flow produced by the system marginal time minimization oriented decision forms an SO (system optimal) state. Such equilibrium states take route travel time-travel price as the only decision variable of travellers' route choice behaviour.

Based on the price-quantity adjustment behaviour principle of the non-Walrasian equilibrium theory in economics, this paper assumes that the travellers' route choice behaviour is not only affected by the travel price signal-travel time but also influenced by the quantity signal, which is considered through the route surplus capacity indicator to reflect the travellers' preference to travel comfort degree. The route surplus capacity is the difference between the route maximum capacity and the route flow, which concerns the physical capacity of the network route and indicates the travel comfort degree. In addition, the route with a larger surplus capacity indicates a higher degree of the route service level, including road infrastructure facilities, environmental satisfaction, and travel fluency. 
When the current network flow is zero, the traveller chooses the route with the largest capacity to experience the most comfortable travel. With the gradual increase of road network flow, the residual capacity on all alternative paths will decrease, and the route travel comfort degree will decrease. Therefore, travellers will adjust their route selection according to the route travel comfort, and the route flow will shift to the alternative path with larger residual capacity. This route adjustment behaviour will lead the traffic flow to a stable state, which is defined as QUE (quantity adjustment user equilibrium) state in this research.

2.2. Mixed Equilibrium. When applying the dual constraint of price and quantity to the route selection behaviour of road network travellers, it means that a group of network travellers take the minimum travel price as the goal, and the traffic flow formed by such travellers' route decision-making behaviour finally stabilises in the price adjustment user equilibrium, that is, Wardrop UE state, while the other group of travellers expect the maximum comfort travel experience, and the traffic flow formed by such travellers' route decision-making behaviour finally stabilises in the QUE state. These two groups of travellers coexist in the road network, so that different decision-making behaviours of route selection interact and influence each other and finally form a UE-QUE mixed equilibrium state. The corresponding dynamic adjustment behaviour of traffic flow is the evolution process of mixed regulation traffic flow.

Given a network $G=(T, A)$, assuming that the travel demand of travellers in the first group following the UE principle is expressed as $d_{w}$, the travel demand of travellers in the second group following the QUE principle is denoted by $\widehat{d}_{w}$. The flows of travellers on route $r \in R_{w}$ in these two groups are represented by $f_{w}^{r}$ and $\widehat{f}_{w}^{r}$, respectively. These route flows are grouped into two vectors and can be expressed by

$$
\left\{\begin{array}{l}
\mathbf{f}=\left(f_{w}^{r}: w \in W, r \in R_{w}\right), \\
\widehat{\mathbf{f}}=\left(\widehat{f}_{w}^{r}: w \in W, r \in R_{w}\right) .
\end{array}\right.
$$

The flow conservation condition is given by

$$
\begin{gathered}
\sum_{r \in R_{w}} f_{w}^{r}=d_{w}, \\
\sum_{r \in R_{w}} \hat{f}_{w}^{r}=\widehat{d}_{w} .
\end{gathered}
$$

The traffic flow on the link $a \in A$ is the aggregated link flow from two groups, which is

$$
x_{a}=\sum_{w \in W} \sum_{r \in R_{w}} \delta_{w}^{r a}\left(f_{w}^{r}+\widehat{f}_{w}^{r}\right), \quad a \in A .
$$

Travellers in the first group take the minimum travel price as the goal, following the UE route rapidity choice behaviour assumption, in which all travellers are supposed to shift to the alternative shorter route to reduce their actual travel time given the current information. The corresponding traffic flow evolution process finally stabilises in the price adjustment user equilibrium, that is, Wardrop UE state. When the traffic network travel demand is low, the route travel time is relatively low, so travellers in the first group select the shortest route with the most rapid time. With an increase in the network travel demand, the route travel time is increased since the traffic demand is gradually assigned to the traffic route network, which evolves to a congested state. Under this circumstance, travellers in the first group are supposed to shift to an alternative route where travel time is less compared with the current route. In addition, the traffic flow will be stable in the equilibrium state where all routes have the same travel time which is equal to the minimum route travel time for the OD pair. This route shift behaviour is predefined as route rapidity choice behaviour, and the formed network equilibrium state is the Wardrop UE state.

In the UE state, the route cost of all used routes between each OD pair is equal to the minimum route cost and less than (or equal to) the other routes with no flows. The equilibrium condition of travellers in the first group following the UE principle is

$$
\left\{\begin{array}{l}
f_{w}^{r}>0, \sum_{a \in A} \delta_{w}^{r a}\left[t_{a}\left(x_{a}\right)\right]=u_{w} \\
f_{w}^{r}=0, \sum_{a \in A} \delta_{w}^{r a}\left[t_{a}\left(x_{a}\right)\right] \geq u_{w} \\
a \in A, r \in R_{w}, w \in W
\end{array}\right.
$$

where $u_{w}$ denotes the minimal travel time between OD pair $w$.

Whereas travellers in the second group expect the maximum comfort travel experience, they follow the QUE route comfort choice behaviour assumption, in which all travellers are supposed to shift to the alternative path with a larger residual capacity to increase their travel comfort degree given the current information. The corresponding traffic flow evolution process finally stabilises in the quantity adjustment user equilibrium, QUE state. When the traffic network travel demand is low, the capacities of all routes are relatively high, and travellers in the second group will select the maximum-surplus-capacity route with the most comfortable travel route. With the increase of travel demand, the surplus capacities of all routes are reduced since the network gradually evolves to a congested state. Under this circumstance, travellers in the second group are supposed to shift to an alternative route where the surplus capacity is greater compared with the current route. In addition, the traffic flow will be stable in the equilibrium state where all route surplus capacities are the same and equal to the maximum surplus capacity of the OD pair. This route shift behaviour is predefined as the route comfort choice behaviour, and the formed network equilibrium state is called the quantity adjustment user equilibrium.

The maximum traffic capacity of the route $r \in R_{w}$ is expressed as

$$
K_{r}=\min \left(\delta_{w}^{r a} K_{a}\right)
$$

The surplus capacity of the route $r \in R_{w}$ is given by

$$
s_{w}^{r}\left(\hat{f}_{w}^{r}\right)=\max \left\{K_{r}-\hat{f}_{w}^{r}, 0\right\} .
$$


The maximum surplus capacity in the OD pair $w$ is defined as

$$
v_{w}=\max _{r \in R_{w}}\left\{s_{w}^{r}\left(\hat{f}_{w}^{r}\right)\right\}
$$

In the QUE state, the route surplus capacities of all used routes between each OD pair are equal to the maximum surplus capacity and greater than (or equal to) the other routes with no flows [51]. The equilibrium condition of travellers in the second group followed the QUE principle is

$$
\left\{\begin{array}{l}
\hat{f}_{w}^{r}>0, s_{w}^{r}\left(\hat{f}_{w}^{r}\right)=v_{w}, \\
\hat{f}_{w}^{r}=0, s_{w}^{r}\left(\hat{f}_{w}^{r}\right) \leq v_{w}, \\
r \in R_{w}, w \in W .
\end{array}\right.
$$

\section{Mixed Equilibrium Switch Model}

The adjustment route flow of route $l$ in OD pair $w$ on day $n$, selected by travellers in the first group, is denoted by $\dot{f}_{w}^{l}(n)=y_{w}^{l}(n)$. Assume that the adjustment route flow of the first group travellers between two alternative routes is proportional to the route cost difference in the dynamical system [24]. When the route cost of the route $k$ is larger than the route cost of route $l$ on time $n: c_{w}^{k}>c_{w}^{l}$, the adjustment flow from route $k$ to $l$ is positive: $y_{w}^{k \longrightarrow l}(n)>0$; conversely, if the route $k$ has lesser cost than the route $l: c_{w}^{k}<c_{w}^{l}$, the corresponding adjustment flow is negative: $y_{w}^{k} \stackrel{w}{\longrightarrow}(n)<0$; and the travellers do not switch if the route costs are equal. Mathematically, the adjustment route flow can be expressed as

$$
\begin{aligned}
& y_{w}^{k \longrightarrow l}(n)=\alpha \cdot \varphi_{w}^{k \longrightarrow l}(c, f(n)), \quad k, l \in R_{w} ; w \in W, \\
& \varphi_{w}^{k \rightarrow l}(c, f(n))= \begin{cases}\frac{\left(c_{k}-c_{l}\right)}{c_{k}} \cdot f_{w}^{k}(n), & \text { if } c_{k}-c_{l} \geq 0, \\
\frac{\left(c_{k}-c_{l}\right)}{c_{l}} \cdot f_{w}^{l}(n), & \text { if } c_{k}-c_{l}<0,\end{cases} \\
& y_{w}^{l}(n)=\sum_{k \neq l} y_{w}^{k \longrightarrow l}(n)=\sum_{k \neq l} \alpha \cdot \varphi_{w}^{k \longrightarrow l}(c, f(n)), \quad k, l \in R_{w} ; w \in W,
\end{aligned}
$$

where $\alpha(0<\alpha<1)$ is the route flow adjustment ratio. Since the right-hand side of equation (9) is continuous, there exists a solution $f_{w}^{l}(n)$ to equation (9) as a well-defined dynamical system. Equation (9) describes the magnitude and direction of the flow switching between two paths. $y_{w}^{l}(n)$ is continuous because it is a summation of $y_{w}^{k} \rightarrow^{l}(n)$.

Likewise, the adjustment route flow of route $l$ in OD pair $w$ on day $n$, ghosen by travellers in the second group, is denoted by $\hat{f}_{w}(n)=\widehat{y}_{w}^{l}(n)$. Assume that the adjustment route flows of the second group travellers are inverse rate with the route surplus capacity difference. When the route surplus capacity of route $k$ is larger than route $l$ on time $n: s_{w}^{k}>s_{w}^{l}$, the adjustment flow from route $k$ to $l$ is negative: $\hat{y}_{w}^{k \stackrel{w}{\longrightarrow} l}(n)<0$; if the route surplus capacity of route $k$ is less than route $l: s_{w}^{k}<s_{w}^{l}$, the corresponding adjustment flow is positive: $\hat{y}_{w}^{k \longrightarrow l}(n)>0$; and the travellers do not switch if the route surplus capacity is equal. Mathematically, the adjustment route flow can be stated as

$$
\begin{aligned}
\hat{y}_{w}^{k-l}(n)=\alpha \cdot \phi_{w}^{k \rightarrow l}(s, \widehat{f}(n)), \quad k, l \in R_{w} ; w \in W, \\
\phi_{w}^{k \rightarrow l}(s, \hat{f}(n))=\left\{\begin{array}{l}
\frac{\left(s_{l}-s_{k}\right)}{s_{l}} \cdot \hat{f}_{w}^{l}(n), \quad \text { if } s_{k}-s_{l} \geq 0, \\
\frac{\left(s_{l}-s_{k}\right)}{s_{k}} \cdot \widehat{f}_{w}^{k}(n), \quad \text { if } s_{k}-s_{l}<0,
\end{array}\right. \\
\hat{y}_{w}^{l}(n)=\sum_{k \neq l} \hat{y}_{w}^{k \rightarrow l}(n)=\sum_{k \neq l} \alpha \cdot \phi_{w}^{k \rightarrow l}(s, \widehat{f}(n)), \quad \alpha>0 ; k, l \in R_{w} ; w \in W .
\end{aligned}
$$

Since the right-hand side of equation (12) is continuous, there exists a solution $\widehat{f}_{w}(n)$ to equation (12) as a well-defined dynamical system. Equation (12) describes the magnitude and direction of the flow switching between two paths. $\hat{y}_{w}^{l}(n)$ is continuous because it is a summation of $\hat{y}_{w}^{k \rightarrow l}(n)$.

Combining equations (11) and (14), we obtain 


$$
\left\{\begin{array}{l}
y_{w}^{l}(n)=\sum_{k \neq l} \alpha \varphi_{w}^{k \longrightarrow l}(c, f(n)), \\
\hat{y}_{w}^{l}(n)=\sum_{k \neq l} \alpha \phi_{w}^{k \longrightarrow l}(s, \widehat{f}(n)), \\
\alpha>0 ; k, l \in R_{w} ; w \in W .
\end{array}\right.
$$

Based on equations (9)-(11), the aggregate route adjustment flow of travellers in the first group on day $n$ can be formulated as

$$
\begin{aligned}
y_{w}(n) & =\sum_{r \in R_{w}} y_{w}^{r}(n) \\
& =\sum_{k, l \in R_{w} \cap k \neq l}\left(y_{w}^{k}(n)+y_{w}^{l}(n)\right) \\
& =\sum_{k, l \in R_{w} \cap k \neq l}\left(\alpha \varphi_{w}^{k \longrightarrow l}(c, f(n))+\alpha \varphi_{w}^{k \longrightarrow l}(c, f(n))\right) \\
& =\left\{\begin{array}{l}
\sum_{k, l \in R_{w} \cap k \neq l}\left(\alpha \frac{\left(c_{l}-c_{k}\right)}{c_{k}} \cdot f_{w}^{k}(n)+\alpha \frac{\left(c_{k}-c_{l}\right)}{c_{k}} \cdot f_{w}^{k}(n)\right)=0, \quad \text { if } c_{k}-c_{l} \geq 0, \\
\sum_{k, l \in R_{w} \cap k \neq l}\left(\alpha \frac{\left(c_{l}-c_{k}\right)}{c_{l}} \cdot f_{w}^{l}(n)+\alpha \frac{\left(c_{k}-c_{l}\right)}{c_{l}} \cdot f_{w}^{l}(n)\right)=0, \quad \text { if } c_{k}-c_{l}<0 .
\end{array}\right.
\end{aligned}
$$

Since $\sum_{r \in R_{w}} y_{w}^{r}(n)=0$, it follows that $\sum_{r \in R_{w}} f_{w}^{r}(n)$ is a constant; hence from $\sum_{r \in R_{w}} f_{w}^{r}(0)=d_{w}$, then $\sum_{r \in R_{w}} f_{w}^{r}(n)=d_{w}$.
According to equations (12)-(14), the summation of the route adjustment flow of travellers in the second group on day $n$ can be expressed by

$$
\begin{aligned}
\hat{y}_{w}(n) & =\sum_{r \in R_{w}} \hat{y}_{w}^{r}(n) \\
& =\sum_{k, l \in R_{w} \cap k \neq l}\left(\hat{y}_{w}^{k}(n)+\hat{y}_{w}^{l}(n)\right) \\
& =\sum_{k, l \in R_{w} \cap k \neq l}\left(\alpha \phi_{w}^{l \longrightarrow k}(s, \hat{f}(n))+\alpha \phi_{w}^{k \longrightarrow l}(s, \hat{f}(n))\right) \\
& =\left\{\begin{array}{l}
\sum_{k, l \in R_{w} \cap k \neq l}\left(\alpha \frac{\left(s_{k}-s_{l}\right)}{s_{l}} \cdot \hat{f}_{w}^{l}(n)+\alpha \frac{\left(s_{l}-s_{k}\right)}{s_{l}} \cdot \hat{f}_{w}^{l}(n)\right)=0, \quad \text { if } s_{k}-s_{l} \geq 0, \\
\sum_{k, l \in R_{w} \cap k \neq l}\left(\alpha \frac{\left(s_{k}-s_{l}\right)}{s_{k}} \cdot \hat{f}_{w}^{k}(n)+\alpha \frac{\left(s_{l}-s_{k}\right)}{s_{k}} \cdot \hat{f}_{w}^{k}(n)\right)=0, \quad \text { if } s_{k}-s_{l}<0,
\end{array}\right.
\end{aligned}
$$

where $\sum_{r \in R_{w}} \hat{f}_{w}^{r}(n)=\sum_{r \in R_{w}} \hat{f}_{w}^{r}(0)=\widehat{d}_{w}$; the flow conservation constraint is satisfied.

Furthermore, the route adjustment flow switching model for a general network with multiple O-D pairs can be expressed as

$$
\left\{\begin{array}{l}
\mathbf{y}(n)=\sum \alpha \cdot \Psi((\mathbf{c}, \mathbf{f}(n)) \\
\widehat{y}(n)=\sum \alpha \cdot \Phi((\mathbf{s}, \widehat{\mathbf{f}}(n))
\end{array}\right.
$$

where 


$$
\begin{aligned}
& \Psi(\mathbf{c}, \mathbf{f}(n))=\left[\begin{array}{cccc}
\Psi_{1}(\mathbf{c}, \mathbf{f}(n)) & 0 & \cdot & 0 \\
0 & \Psi_{2}(\mathbf{c}, \mathbf{f}(n)) & 0 & \cdot \\
\cdot & 0 & \cdot & 0 \\
0 & \cdot & 0 & \Psi_{w}(\mathbf{c}, \mathbf{f}(n))
\end{array}\right], \\
& \Phi(\mathbf{s}, \widehat{\mathbf{f}}(n))=\left[\begin{array}{cccc}
\Phi_{1}(\mathbf{s}, \widehat{\mathbf{f}}(n)) & 0 & \cdot & 0 \\
0 & \Phi_{2}(\mathbf{s}, \widehat{\mathbf{f}}(n)) & 0 & \cdot \\
\cdot & 0 & \cdot & 0 \\
0 & \cdot & 0 & \Phi_{w}(\mathbf{s}, \widehat{\mathbf{f}}(n))
\end{array}\right],
\end{aligned}
$$

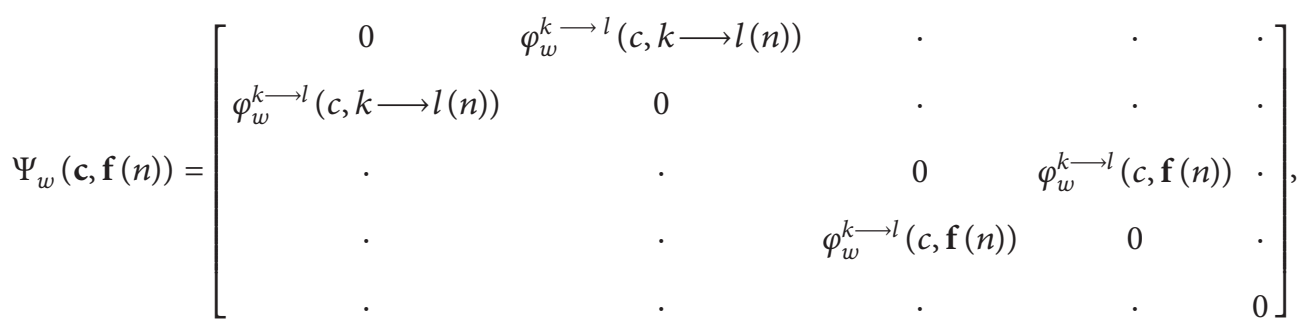

$$
\begin{aligned}
& \Phi_{w}(\mathbf{s}, \widehat{\mathbf{f}}(n))=\left[\begin{array}{ccccc}
0 & \phi_{w}^{k \longrightarrow l}(s, \widehat{\mathbf{f}}(n)) & \cdot & \\
\phi_{w}^{k \longrightarrow l}(s, \widehat{\mathbf{f}}(n)) & 0 & \cdot & \cdot & \cdot \\
\cdot & . & 0 & \phi_{w}^{k \longrightarrow l}(s, \widehat{f}(n)) & \cdot \\
\cdot & . & \phi_{w}^{k \longrightarrow l}(s, \widehat{\mathbf{f}}(n)) & 0 & \cdot \\
. & . & . & . & 0
\end{array}\right] . \\
& \left\{\begin{array}{l}
\mathbf{f}(n+1)=\mathbf{f}(n)+\mathbf{y}(n) \\
\widehat{\mathbf{f}}(n+1)=\widehat{\mathbf{f}}(n)+\widehat{y}(n) \\
\mathbf{f}(0)=\mathbf{f}^{(0)} \\
\widehat{\mathbf{f}}(0)=\widehat{\mathbf{f}}^{(0)}
\end{array}\right.
\end{aligned}
$$

Each element $\left(y_{w}^{l}(n)\right.$ and $\left.\hat{y}_{w}^{l}(n)\right)$ in system (18) is continuous. Consequently, the solution existence for system (18) is guaranteed.

Therefore, the route flow of the first group in route $l$ on day $n+1$ is

$$
f_{w}^{l}(n+1)=f_{w}^{l}(n)+\sum_{k \longrightarrow l} \alpha \varphi_{w}^{k \longrightarrow l}(c, f(n)) .
$$

And the route flow of the second group in route $l$ on day $n+1$ can be stated as

$$
\widehat{f}_{w}^{l}(n+1)=\hat{f}_{w}^{l}(n)+\sum_{k \neq l} \alpha \phi_{w}^{k \longrightarrow l}(s, \widehat{f}(n)) .
$$

Then, the adjustment route flow switching model can be restated as

$$
\begin{aligned}
& f_{w}^{l}(n+1)=f_{w}^{l}(n)+\sum_{k \neq l} \alpha \varphi_{w}^{k \longrightarrow l}(c, f(n)), \\
& \hat{f}_{w}^{l}(n+1)=\hat{f}_{w}^{l}(n)+\sum_{k \neq l} \alpha \phi_{w}^{k \longrightarrow l}(s, \hat{f}(n)) .
\end{aligned}
$$

Combining equations (18), (21a), and (21b), given the initial conditions: $(n \in[0, N]), \quad \mathbf{f}(n=0)=\mathbf{f}^{0} \quad$ and $\widehat{\mathbf{f}}(n=0)=\widehat{\mathbf{f}}^{0}$, the mixed equilibrium switch model is given by

\section{Property Analyse}

4.1. Equivalence between Stable State and Mixed Equilibrium Condition. In the present section, we establish a mixed equilibrium switch model, and if it reaches a stable point $\left(\mathbf{f}^{*}, \widehat{\mathbf{f}}^{*}\right)$, which means

$$
\left\{\begin{array}{l}
\mathbf{f}^{*}(n+1)=\mathbf{f}^{*}(n), \\
\widehat{\mathbf{f}}^{*}(n+1)=\widehat{\mathbf{f}}^{*}(n),
\end{array}\right.
$$

then, it follows that all the adjustment route flow is zero:

$$
\left\{\begin{array}{l}
\mathbf{y}^{*}(n)=\sum \alpha \Psi\left(\mathbf{c}, \mathbf{f}^{*}(n)\right)=0, \\
\widehat{y}^{*}(n)=\sum \alpha \Phi\left(\mathbf{s}, \widehat{f}^{*}(n)\right)=0 .
\end{array}\right.
$$

Assume that the route $l$ is the shortest route with the minimum route travel cost in OD pair $w$, stated as

$$
c_{w}^{k} \geq c_{w}^{l}=u_{w}>0, \quad k \neq l \in R_{w} .
$$


Substituting equations (10) into (24), we have

$$
y_{w}^{l *}=\sum_{k \neq l} \alpha \varphi_{w}^{k \rightarrow l}(c, f(n))=\sum_{k \neq l} \alpha \frac{\left(c_{k}-c_{l}\right)}{c_{k}} \cdot f_{w}^{k}(n)=0 .
$$

The route flow is nonnegative, and so, there is $f_{w}^{k}(n) \geq 0, k \in R_{w}, w \in W$; combining it with equations (25) and (26) we get

$$
\left\{\begin{array}{l}
f_{w}^{k}>0, c_{w}^{k}=c_{w}^{l}=u_{w} \\
f_{w}^{k}=0, c_{w}^{k}>c_{w}^{l}=u_{w} \\
k, l \in R_{w}, w \in W
\end{array}\right.
$$

Likewise, assuming that the route $l$ is the most comfortable route with the maximum surplus capacity in the OD pair $w$, then

$$
s_{w}^{l}=v_{w} \geq s_{w}^{k} \geq 0, \quad k \neq l \in R_{w} .
$$

Substituting equations (13) into (24), we have

$$
\widehat{y}_{w}^{l *}=\sum_{k \neq l} \alpha \phi_{w}^{k \longrightarrow l}(s, \hat{f}(n))=\sum_{k \neq l} \alpha \frac{\left(s_{l}-s_{k}\right)}{s_{k}} \cdot \hat{f}_{w}^{k}(n)=0 .
$$

With the nonnegative property of route flow, which is $\widehat{f}_{w}^{k} \geq 0, k \in R_{w}, w \in W$, and combining it with equations (28) and (29), it then follows that

$$
\left\{\begin{array}{l}
\hat{f}_{w}^{k}>0, \quad s_{w}^{k}=s_{w}^{l}=v_{w} \\
\hat{f}_{w}^{k}=0, \quad s_{w}^{k} \leq s_{w}^{l}=v_{w} \\
k, l \in R_{w}, \quad w \in W
\end{array}\right.
$$

It can be seen that equations (27) and (30) are actually the equilibrium conditions of the travellers in two groups, which shows that the stable point $\left(\mathbf{f}^{*}, \widehat{\mathbf{f}}^{*}\right)$ of the proposed switch model is equivalent to the mixed equilibrium condition.

4.2. Uniqueness of Solution. Based on the variational inequality (VI) theory, it is clear that the mixed equilibrium switch model (22) can be rewritten as the following VI problem:

$$
\begin{aligned}
F\left(\mathbf{f}^{*}, \widehat{\mathbf{f}}^{*}\right)^{\mathrm{T}} \cdot\left(\begin{array}{c}
\mathbf{f}-\mathbf{f}^{*} \\
\widehat{\mathbf{f}}-\widehat{\mathbf{f}}^{*}
\end{array}\right)= & \sum \sum \alpha \cdot \Psi\left(\mathbf{c}, \mathbf{f}^{*}\right) \cdot\left(\mathbf{f}-\mathbf{f}^{*}\right) \\
& +\sum \sum \alpha \cdot \Phi\left(\mathbf{s}, \widehat{\mathbf{f}}^{*}\right) \cdot\left(\widehat{\mathbf{f}}-\widehat{\mathbf{f}}^{*}\right) \\
\geq & 0 .
\end{aligned}
$$

We note that it is known that if the function $F(\mathbf{f}, \widehat{\mathbf{f}})$ is strictly monotone with $(\mathbf{f}, \widehat{\mathbf{f}})$, then there exists a unique model solution.

Proof. Assume that $\left(\mathbf{f} /, \widehat{\mathbf{f}}^{\prime}\right)$ and $\left(\mathbf{f}^{*}, \widehat{\mathbf{f}}^{*}\right)$ are solutions to the VI problem $(31)$, and $\left(\mathbf{f}, \widehat{\mathbf{f}}^{\prime}\right) \neq\left(\left(\mathbf{f}^{*}, \widehat{\mathbf{f}}^{*}\right)\right.$, so that

$$
\sum\left(\sum \alpha \cdot \Psi(\mathbf{c}, \mathbf{f} \prime)\right) \cdot(\mathbf{f}-\mathbf{f} \prime)+\sum \sum \alpha \cdot \Phi\left(\mathbf{s}, \widehat{\mathbf{f}}^{\prime}\right) \cdot\left(\widehat{\mathbf{f}}-\widehat{\mathbf{f}}^{\prime}\right) \geq 0,
$$

$$
\sum\left(\sum \alpha \cdot \Psi\left(\mathbf{c}, \mathbf{f}^{*}\right)\right) \cdot\left(\mathbf{f}-\mathbf{f}^{*}\right)+\sum\left(\sum \alpha \cdot \Phi\left(\mathbf{s}, \widehat{\mathbf{f}}^{*}\right)\right) \cdot\left(\widehat{\mathbf{f}}-\widehat{\mathbf{f}}^{*}\right) \geq 0 .
$$

Let $(\mathbf{f}, \widehat{\mathbf{f}})=\left(\mathbf{f}^{*}, \widehat{\mathbf{f}}^{*}\right)$ in equation (32) and $(\mathbf{f}, \widehat{\mathbf{f}})=\left(\mathbf{f} /, \widehat{\mathbf{f}}^{\prime}\right)$ in equation (33), and adding the resulting inequalities, one obtains

$$
\left(\begin{array}{l}
\sum\left[\left(\sum \alpha \cdot \Psi(\mathbf{c}, \mathbf{f} \prime)\right)-\left(\sum \alpha \cdot \Psi\left(\mathbf{c}, \mathbf{f}^{*}\right)\right)\right]^{\mathrm{T}} \\
\sum\left[\left(\sum \alpha \cdot \Phi(\mathbf{s}, \mathbf{f} \prime)\right)-\left(\sum \alpha \cdot \Phi\left(\mathbf{s}, \widehat{\mathbf{f}}^{*}\right)\right)\right]^{\mathrm{T}}
\end{array}\right)^{\mathrm{T}} \cdot\left(\begin{array}{c}
\mathbf{f}^{*}-\mathbf{f} \prime \\
\widehat{\mathbf{f}}^{*}-\widehat{\mathbf{f}}^{\prime}
\end{array}\right) \geq 0 .
$$

Apparently, the inequality (34) is in contradiction to the definition of strict monotonicity. Hence $\left(\mathbf{f}, \widehat{\mathbf{f}}^{\prime}\right)=\left(\mathbf{f}^{*}, \widehat{\mathbf{f}}^{*}\right)$; the uniqueness of the solution holds.

4.3. Stability of Solution. LaSalle's theorem is used to address the stability properties of the mixed equilibrium switch model. The candidate Lyapunov function [11] that we applied for the travellers of the first group is

$$
V(f(n))=\int_{0}^{x(n)} t(\eta) \mathrm{d} \eta
$$

It represents the objective function of the UE assignment. Since $x(n)$ is a linear transformation of $f(n)$ from equation (3), $\|f(n)\| \longrightarrow \infty,\|x(n)\| \longrightarrow \infty$, and $t(x(n))$ is a monotonically increasing function of $x(n)$. Hence, $f(n) \longrightarrow \infty, \int_{0}^{x(n)} t(\eta) \mathrm{d} \eta \longrightarrow \infty$ and $\lim _{\|f(n)\| \longrightarrow \infty} V$ $(f(n))=\infty$, which means that $V(f(n))$ is a continuously differentiable, positive definite radially unbounded function. Equation (35) can be expanded as

$$
\begin{aligned}
V(f(n)) & =\int_{0}^{x(n)} t(\eta) \mathrm{d} \eta=\int_{n^{0}}^{n} t(\eta) \frac{\mathrm{d} \eta}{\mathrm{d} n} \mathrm{~d} n \\
& =\int_{n^{0}}^{n} t(\eta) \frac{\mathrm{d} \eta}{\mathrm{d} f} \frac{\mathrm{d} f}{\mathrm{~d} n} \mathrm{~d} n .
\end{aligned}
$$

Here, $n^{0}$ is the initial time in the dynamical switch system corresponding to the initial state $f(0), n \prime$ is the time in the dynamical switch system when the system reaches the state $f(n)$. Equation (36) converts equation (35) to an integral along a route in the plane parameterized by $n$. The conversion is to avoid dealing with the complex interactions among $x(n)$, which is caused by the different classes of route choice behaviour principle in this research. And so the derivative of the Lyapunov function along the trajectories of the switch process is

$$
\dot{V}(f(n))=\frac{\mathrm{d} V}{\mathrm{~d} n}(f(n))=\frac{d\left(\int_{n^{0}}^{h} t(\eta)(\mathrm{d} \eta / \mathrm{d} f)(\mathrm{d} f / \mathrm{d} n) \mathrm{d} n\right)}{\mathrm{d} t},
$$

where $(\mathrm{d} \eta / \mathrm{d} f)=(\mathrm{d} x / \mathrm{d} f)=(\mathrm{d}(\delta f) / \mathrm{d} f)=\delta, t(\eta)(\mathrm{d} \eta / \mathrm{d} f)$ $=t(\eta) \delta=c$, and $(\mathrm{d} f / \mathrm{d} n)=\dot{f}=y$. Therefore, 


$$
\begin{aligned}
\dot{V}(f(n)) & =\frac{d\left(\int_{n^{0}}^{h} t(\eta)(\mathrm{d} \eta / \mathrm{d} f)(\mathrm{d} f / \mathrm{d} n) \mathrm{d} n\right)}{\mathrm{d} t} \\
& =\mathbf{c} \cdot \mathbf{y}(n)=\mathbf{c} \cdot \sum \alpha \Psi(\mathbf{c}, \mathbf{f}(n)) .
\end{aligned}
$$

Now, from our earlier remark in Section 4.1, if and only if the dynamic switch process reaches the mixed equilibrium state, condition $\left\{\begin{array}{l}c_{k}>c^{*}, f_{w}^{k}=0 \\ c_{k}=c^{*}, f_{w}^{k}>0\end{array}\right.$ holds and $\dot{V}\left(f^{*}(n)\right)=\mathbf{c}^{*}$. $\sum \alpha \Psi^{*}(\mathbf{c}, \mathbf{f}(n))=0$. Then, $c>0, \quad \alpha>0, \quad f(n) \geq 0$, and $\Psi(\mathbf{c}, \mathbf{f}(n))$ is not positively invariant, so $\dot{V}(f(n))$ is nonpositive definite.

The candidate Lyapunov function [25] that we applied for the travellers of the second group is

$$
W(\hat{f}(n))=\sum \hat{f}(n) \cdot(v-s) .
$$

Apparently, the surplus capacity decreases with the increase of network route flow, that is, $\widehat{f}(n) \uparrow, s \downarrow,(v-s) \uparrow$. Hence $\hat{f}(n) \longrightarrow \infty, \sum \hat{f}(n) \cdot(v-s) \longrightarrow \infty$ and $\lim _{\widehat{\|}(n) \| \rightarrow \infty} W(\widehat{f}(n))=\infty$, which means that $W(\widehat{f}(n))$ is a continuously differentiable, positive definite radially unbounded function. Equation (39) can be rewritten as

$$
\begin{aligned}
\dot{W}(\hat{f}(n)) & =\frac{\mathrm{d} W}{\mathrm{~d} n}(\widehat{f}(n))=\frac{\mathrm{d} W}{\mathrm{~d}(\widehat{f}(n))}(\widehat{f}(n)) \cdot \frac{\mathrm{d}(\hat{f}(n))}{\mathrm{d} n} \\
& =\frac{\mathrm{d} W}{\mathrm{~d}(\hat{f}(n))}(\hat{f}(n)) \cdot \hat{y}(n),
\end{aligned}
$$

where

$$
\begin{aligned}
\frac{\mathrm{d} W(\widehat{f}(n))}{\mathrm{d}(\hat{f}(n))} & =\left[\frac{\partial W(\hat{f}(n))}{\partial\left(\hat{f}_{1}(n)\right)} \frac{\partial W(\hat{f}(n))}{\partial\left(\hat{f}_{2}(n)\right)} \cdots \frac{\partial W(\hat{f}(n))}{\partial\left(\hat{f}_{r}(n)\right)}\right] \\
& =\left[\begin{array}{llll}
-s_{1} & -s_{2} & \cdots & -s_{r}
\end{array}\right]=-\mathbf{s},
\end{aligned}
$$

and so,

$$
\dot{W}(\hat{f}(n))=-\mathbf{s} \cdot \mathbf{y}(n)=-\mathbf{s} \cdot \sum \alpha \Phi(\mathbf{s}, \widehat{f}(n)) .
$$

If and only if the dynamic switch process reaches the mixed equilibrium state, condition $\left\{\begin{array}{l}s_{k}<s^{*}, \widehat{f}_{w}^{k}=0 \\ s_{k}=s^{*}, \widehat{f}_{w}^{k}>0\end{array}\right.$ holds, and $\dot{W}\left(\widehat{f}^{*}(n)\right)=-\mathbf{s}^{*} \cdot \sum \alpha \Phi^{*}(\mathbf{s}, \hat{f}(n))=0$. Then, $s \geq 0$, $\alpha>0, \widehat{f}(n) \geq 0$, and $\Phi(\mathbf{s}, \widehat{f}(n))$ is not positively invariant, so $\dot{W}(\hat{f}(n))$ is nonpositive definite too.

Above all, the dynamic switch model (22) is stable and the solution of the dynamic switch model (22) converges to the mixed equilibrium state.

\section{Numerical Example}

In this subsection, we study the performance of the proposed dynamic traffic flow adjustment model, which synthetically considers the travellers with a different route choice behaviour principle. The tested network is shown in Figure 1 [25]. The

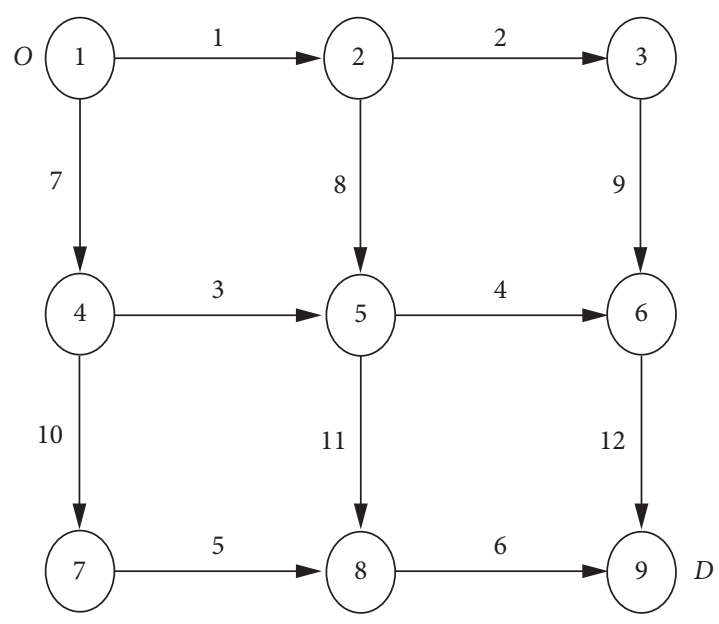

Figure 1: Test traffic network.

TABLE 2: The parameters in the link travel time functions.

\begin{tabular}{lcc}
\hline Link & $t_{a}^{0}$ & $c_{a}$ \\
\hline 1 & 8 & 63 \\
2 & 16 & 60 \\
3 & 6 & 74 \\
4 & 12 & 65 \\
5 & 10 & 60 \\
6 & 10 & 68 \\
7 & 12 & 68 \\
8 & 14 & 71 \\
9 & 8 & 60 \\
10 & 6 & 66 \\
11 & 7 & 64 \\
12 & 8 & 67 \\
\hline
\end{tabular}

traffic demand pattern between the OD pairs is assumed to be $D=240$; assuming that $50 \%$ of the travellers follow the quantity adjustment principle, $\beta=50 \%$, then the traffic demand of the first group and the second group is $d=120$ and $\widehat{d}=120$. The route adjustment flow ratio is assumed as $\alpha=0.6$.

The incidence matrix of routes and links for the network is given in equation (43), and a simplified link travel time function that is often used in practice developed by the U.S. BPR (Bureau of Public Roads) is listed as follows, with freeflow travel time and link capacity given in Table 2:

$$
\Delta^{\mathrm{T}}=\left[\begin{array}{llllllllllll}
1 & 1 & 0 & 0 & 0 & 0 & 0 & 0 & 1 & 0 & 0 & 1 \\
1 & 0 & 0 & 1 & 0 & 0 & 0 & 1 & 0 & 0 & 0 & 1 \\
1 & 0 & 0 & 0 & 0 & 1 & 0 & 1 & 0 & 0 & 1 & 0 \\
0 & 0 & 1 & 1 & 0 & 0 & 1 & 0 & 0 & 0 & 0 & 1 \\
0 & 0 & 1 & 0 & 0 & 1 & 1 & 0 & 0 & 0 & 1 & 0 \\
0 & 0 & 0 & 0 & 1 & 1 & 1 & 0 & 0 & 1 & 0 & 0
\end{array}\right],
$$

$$
t_{a}\left(x_{a}\right)=t_{a}^{0}\left[1+0.15\left(\frac{x_{a}}{c_{a}}\right)^{4}\right], \quad \forall a \in A
$$




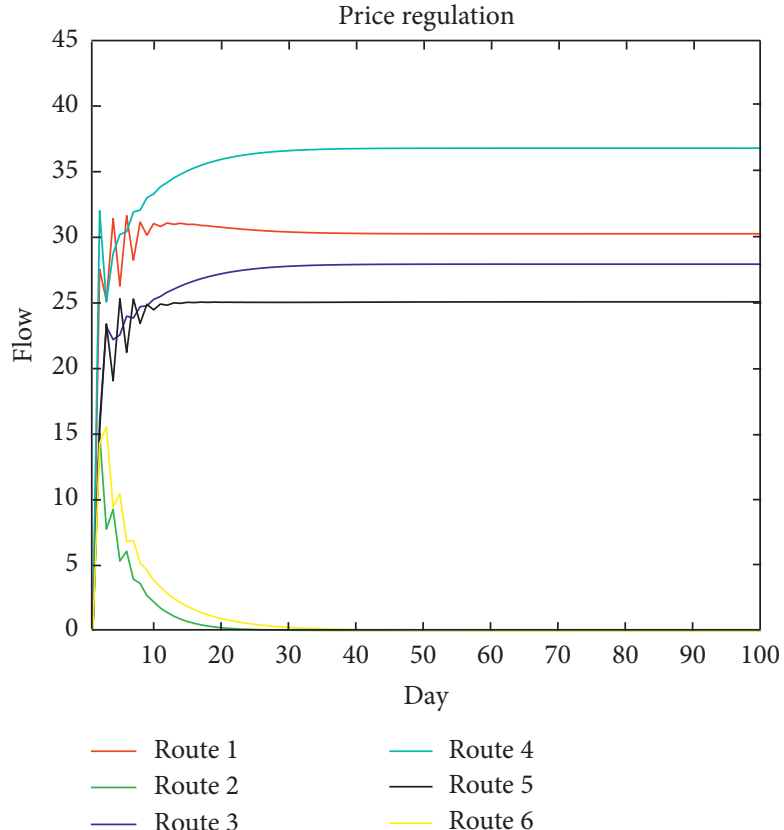

(a)

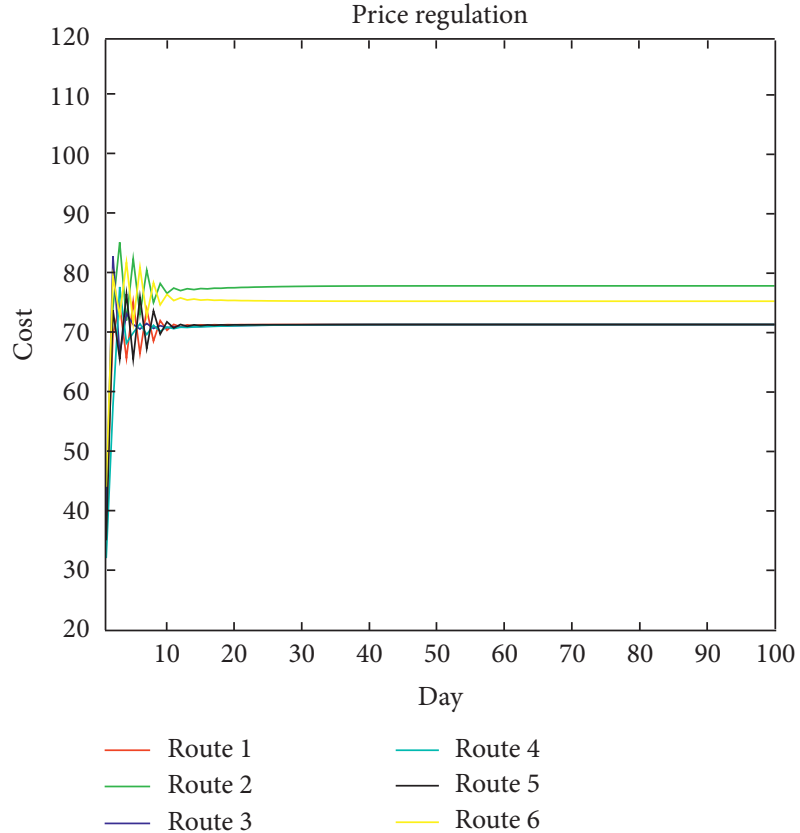

(b)

Figure 2: Evolutionary trajectories of route flows (a) and route travel costs (b) for travellers in the first group.

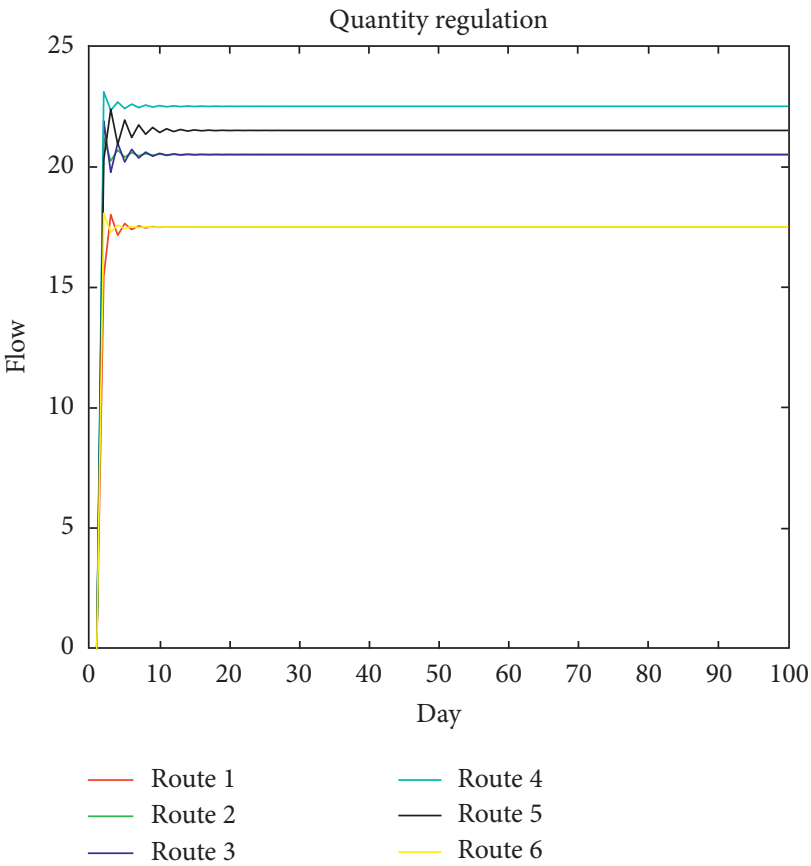

(a)

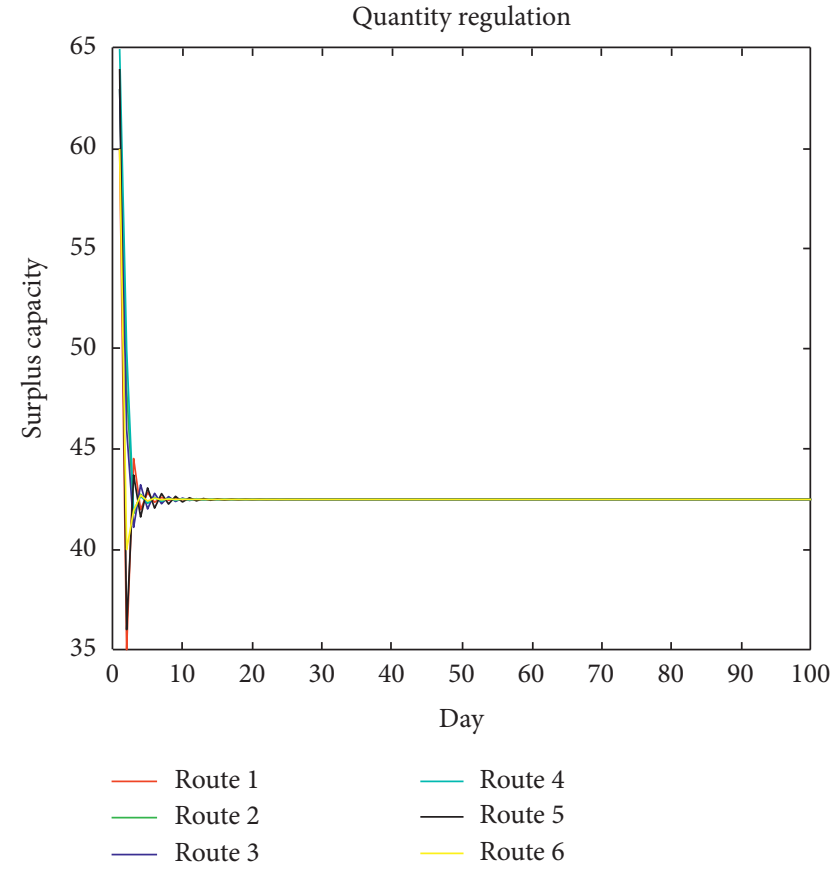

(b)

FIGURE 3: Evolutionary trajectories of route flows (a) and route surplus capacity (b) for travellers in the second group.

Figure 2 shows the evolution process of route flows and correspondent route travel time for travellers in the first group, while Figure 3 depicts the evolution process of route flows and correspondent route surplus capacity for travellers in the second group. Meanwhile, the route flow pattern and the corresponding route travel time in the mixed equilibrium state for travellers in the first group are tabulated in Table 3 as $f$ and $c$, and the route flow pattern and the corresponding route surplus capacity in the mixed equilibrium state for travellers in the second group are tabulated in Table 3 as $\hat{f}$ and $s$.

By combining Figures 2 and 3 with Table 3 , it shows that the specific evolution process wherein the traffic flows 
TABLE 3: Route flow, route travel cost, and surplus capacity in mixed equilibrium for travellers.

\begin{tabular}{|c|c|c|c|c|c|}
\hline Route & Link & $f$ & $c$ & $\widehat{f}$ & $s$ \\
\hline 1 & $1,2,9,12$ & 30.25 & 71.30 & 17.50 & 42.50 \\
\hline 2 & $1,4,8,12$ & 0.00 & 77.81 & 20.50 & 42.50 \\
\hline 3 & $1,6,8,11$ & 27.93 & 71.30 & 20.50 & 42.50 \\
\hline 4 & $3,4,7,12$ & 36.77 & 71.30 & 22.50 & 42.50 \\
\hline 5 & $3,6,7,11$ & 25.06 & 71.30 & 21.50 & 42.50 \\
\hline 6 & $5,6,7,10$ & 0.00 & 75.23 & 17.50 & 42.50 \\
\hline
\end{tabular}
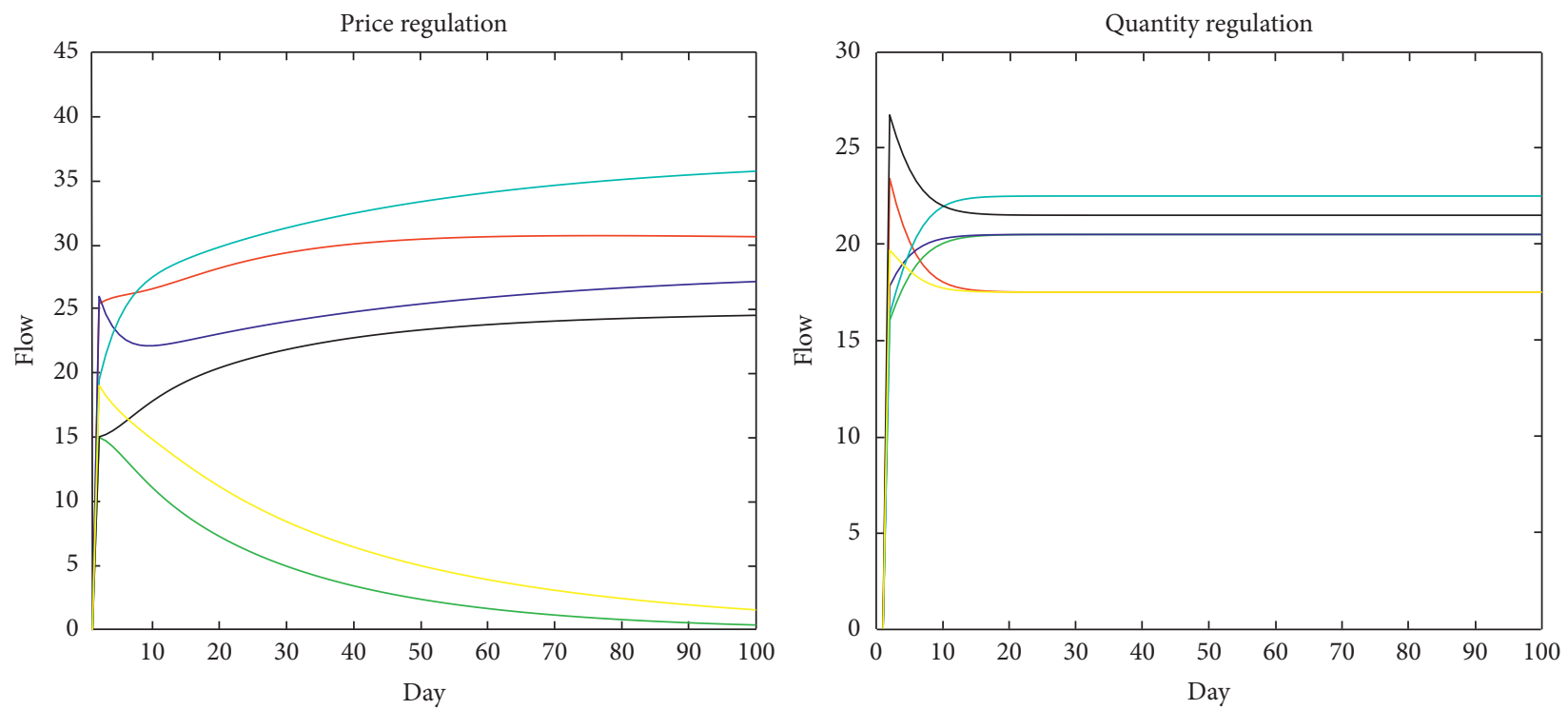

(a)
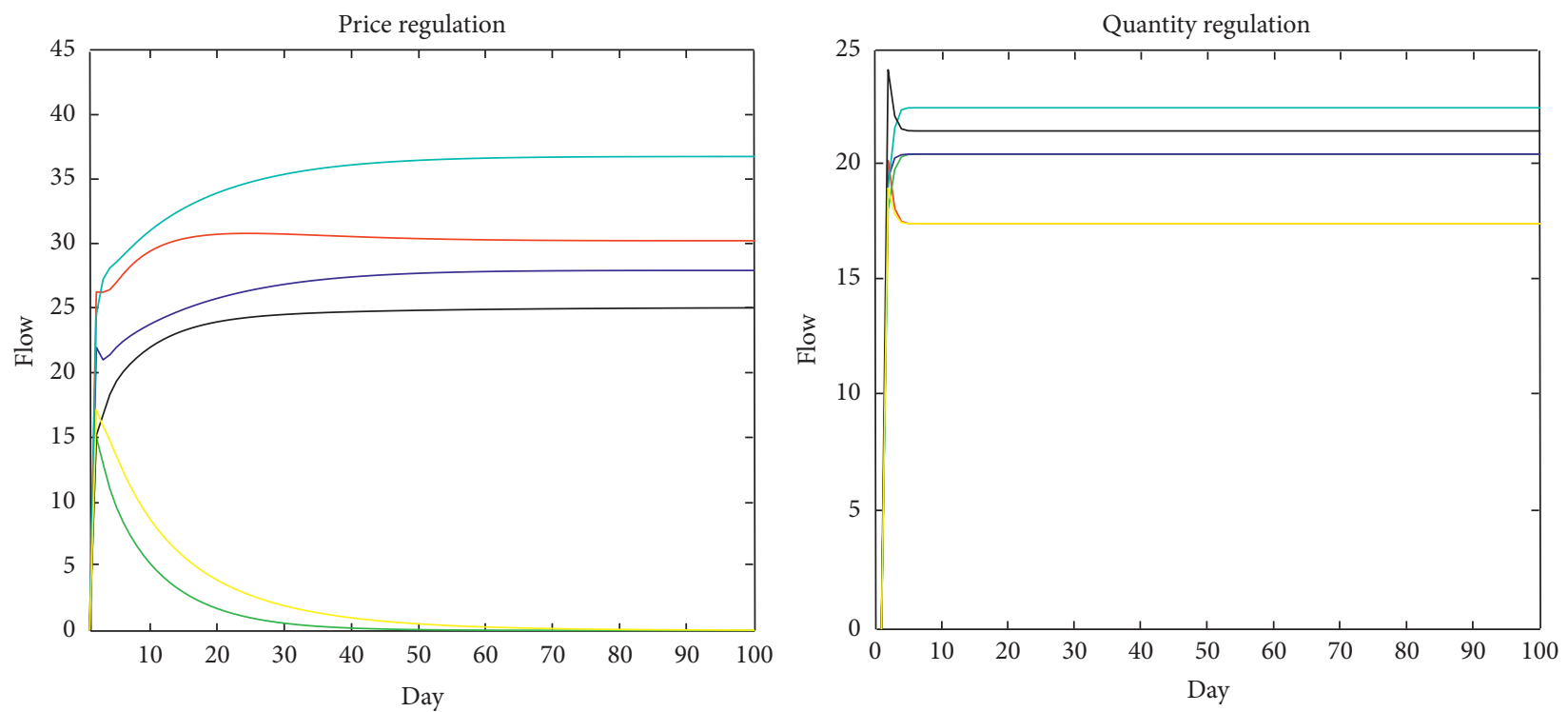

(b)

FIgURE 4: Continued. 

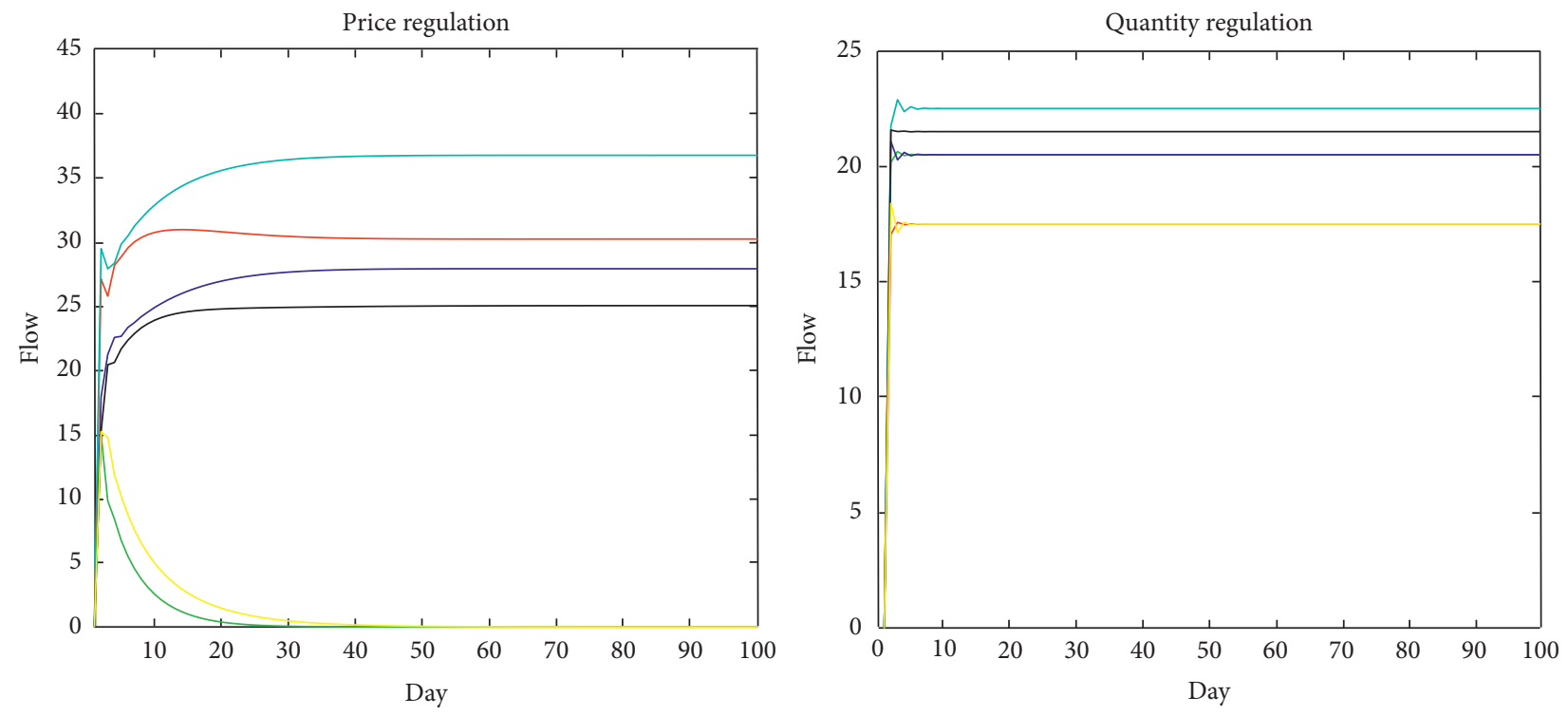

(c)

Figure 4: Flow evolution of test traffic network with different traffic flow proportional adjustment ratios. (a) $\alpha=0.1$. (b) $\alpha=0.3$. (c) $\alpha=0.5$.

gradually converges to the equilibrium state after a fluctuant period. The above observation indicates that travellers in the first group, following UE principle, will evolve to a UE state, in which the travel costs of all used routes between the same OD pairs are equal and minimal, and travellers in the second group, following QUE principle, will evolve to a quantityadjusted user equilibrium state in which the route surplus capacities of all used routes between the same OD pairs are equal and maximum.

Next, we test the performance of our mixed equilibrium switch model under different small constant flow adjustment ratios. Figures 4(a)-4(c) depict the evolution processes of flows in the mixed equilibrium state for travellers in the first group and the second group when the adjustment ratio is set to be $0.1,0.3$, and 0.5 , respectively. It shows that, with different initial traffic flow patterns and route adjustment ratios, the dynamic system shares the same final equilibrium traffic flow pattern, which further testifies the uniqueness and stability of solution for the proposed mixed equilibrium switch model.

Parameter $\alpha$ represents the proportion of travellers who reconsider their routes in the next day according to the current network information, where the travellers in the first group shift to the route with lesser route travel time and the travellers in the second group switch to the route with higher route surplus capacity to experience more comfortable travel. Figure 4 shows that, with the increased value of $\alpha$, the number of travellers who adjust their route choice is increased. This shows that a larger $\alpha$ will make the corresponding trajectory smoother and steadier and the fluctuation smaller. This means that, with an increase in the number of travellers who adjust their current route choice in accordance with the network information during the evolution process, the total benefit achieved by the route adjustment decision increases, which encourages travellers to shift to the equilibrium state. Therefore, the convergent speed is accelerated, and the evolution process from disequilibrium to equilibrium is shortened.

Clearly, from the above results, we can observe that traffic flows all converge to the stable state after a fluctuant period and that the proposed route adjustment switch model simulates the ideal traffic flow evolution of the two groups of travellers' route choice behaviour. Table 3 shows that, for travellers in the first group, the demand is entirely loading in the routes with the minimum travel times in the stable state, and the flows in the second group are stable when all of the surplus capacities of the routes are equal. Hence, the stable state is exactly the mixed equilibrium state formed by the different behaviours of these two groups of travellers. From Figure 4, we find that a smaller flow adjustment ratio will make the corresponding trajectory converge to the equilibrium slower, which is caused by the inactive route adjustment behaviour of travellers. And the evolution process under the quantity regulation is always smoother compared with the one under the price regulation with the same other condition, which is mainly caused by the different perception degree of price signal and quantity signal in the traffic network. In general, the travel surplus capacity is more visualised than the travel cost, so that the quantity adjustment behaviour is a universal phenomenon that should not be neglected in the areas of travel behaviour analysis.

\section{Conclusions}

With the rapid development of science and technology and the urbanisation process, new traffic patterns have emerged from large-scale urban infrastructure construction, road network expansion, and so on. To rationally characterise the traffic flow dynamic evolution process from disequilibrium to equilibrium, we studied the new quantity adjustment user 
equilibrium criterion and the classic user equilibrium criterion based on the price-quantity adjustment behaviour principle of the non-Walrasian equilibrium theory. This paper categorises all travellers into two groups according to their route choice behaviour and simulates the dynamic evolution process of the interacted traffic flow. Thus, a proportional-switch adjustment model is established to reflect the route adjustment behaviour interaction between the traditional UE travellers and the QUE travellers, which converged to a mixed equilibrium state. This mixed equilibrium not only considers the diversity of the route selection criteria of the travellers but also elaborates on the interaction between the different travellers' groups. This deepens the understanding of network traffic flows and improves the level of urban traffic planning and management.

This work opens up several opportunities for further research, including (1) the possibility of ascertaining the proportional relationship of different traveller groups in mixed equilibrium, (2) possibilities to devise the appropriate forms defining the different route comfort and the corresponding traffic flow equilibrium conditions, and (3) the extension of the decision factors in route choice process, such as the inherent preference, previous experience, and social interaction of transportation information.

\section{Notations}

\begin{tabular}{|c|c|}
\hline$T:$ & Set of nodes \\
\hline$A:$ & Set of links, where $a \in A$ \\
\hline$W:$ & $\begin{array}{l}\text { Set of origin-destination pairs, } \\
\text { where } w \in W\end{array}$ \\
\hline$R_{w}:$ & $\begin{array}{l}\text { Set of routes in OD pair } w \text {, where } \\
r, k, l \in R_{w}\end{array}$ \\
\hline$c_{a}:$ & Traffic flow on the link $a \in A$ \\
\hline$t_{a}=t_{a}\left(x_{a}\right)$ & $\begin{array}{l}\text { Travel time function on link } \\
a \in A\end{array}$ \\
\hline$\delta_{w}^{r a}:$ & $\begin{array}{l}\text { Element of link-route incidence } \\
\text { matrix, where } \delta_{w}^{r a}=1 \text { if the route } \\
r \in R_{w} \text { contains link } a \text {, and } 0 \\
\text { otherwise }\end{array}$ \\
\hline$d_{w}:$ & $\begin{array}{l}\text { Travel demand of travellers in } \\
\text { the first group }\end{array}$ \\
\hline$\widehat{d}_{w}:$ & $\begin{array}{l}\text { Travel demand of travellers in } \\
\text { the second group }\end{array}$ \\
\hline$f_{w}^{r}:$ & $\begin{array}{l}\text { Traffic flows of travellers in the } \\
\text { first group on route } r \in R_{w}\end{array}$ \\
\hline$J_{u}$ & $\begin{array}{l}\text { Traffic flows of travellers in the } \\
\text { second group on route } r \in R_{w}\end{array}$ \\
\hline $\mathbf{f}=\left(f_{w}^{r}: w \in W, r \in R_{w}\right):$ & $\begin{array}{l}\text { Set of route flows of travellers in } \\
\text { the first group }\end{array}$ \\
\hline$\widehat{\mathbf{f}}=\left(\widehat{f}_{w}^{r}: w \in W, r \in R_{w}\right):$ & $\begin{array}{l}\text { Set of route flows of travellers in } \\
\text { the second group }\end{array}$ \\
\hline & Travel time of route $r \in R_{w}$ \\
\hline 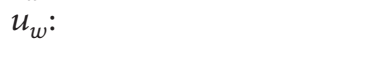 & $\begin{array}{l}\text { Minimal travel time between OD } \\
\text { pair } w\end{array}$ \\
\hline . & $\begin{array}{l}\text { Maximum traffic capacity of the } \\
\text { route } r \in R_{w}\end{array}$ \\
\hline$s_{w}^{r}$ & $\begin{array}{l}\text { Surplus capacity of the route } \\
r \in R_{w}\end{array}$ \\
\hline
\end{tabular}

$v_{w}: \quad$ Maximum surplus capacity in OD pair

$y_{w}^{l}(n): \quad \quad$ Adjustment route flow of travellers in the first group on the route $l$ in OD pair $w$ at day $n$ Adjustment route flow of travellers in the second group on the route $l$ in OD pair $w$ at day $n$

$\mathbf{y}=\left(y_{w}^{l}: w \in W, l \in R_{w}\right)$ : Set of adjustment route flows of travellers in the first group

$\widehat{y}=\left(\widehat{y}_{w}^{l}: w \in W, l \in R_{w}\right)$ : Set of adjustment route flows of travellers in the second group

$\alpha(0<\alpha<1)$ : $\quad$ Route flow adjustment ratio

$\beta$ : Proportion of the travellers in the second group follows the quantity adjustment principle $\varphi_{w}^{k} \rightarrow l: \quad \quad$ Adjustment flow function of travellers in the first group from route $k$ to $l$ in OD pair $w$

$\phi_{w}^{k \longrightarrow l}:$ Adjustment flow function of travellers in the second group from route $k$ to $l$ in OD pair $w$ $n:$ The dynamical system time unit that relates only to the solution procedure and has no mapping to the real system, which is defined as a day in this paper.

\section{Data Availability}

The data used to support the findings of this study are available from the corresponding author upon request.

\section{Conflicts of Interest}

The authors declare that there are no conflicts of interest regarding the publication of this paper.

\section{Acknowledgments}

This work was supported by the National Natural Science Foundation of China under Grant nos. 51338002, 51978082, and 51808057. This work was in part supported by Hunan Provincial Natural Science Foundation of China (no. 2019JJ30026), the Young Elite Scientists Sponsorship Program by Hunan Province of China (2018RS3074), Changsha Science and Technology Bureau Project (kq1801056), Open Fund of Hunan Key Laboratory of Smart Roadway and Cooperative Vehicle-Infrastructure Systems (kfj180702 and kfj190702), Innovation Team Project for Transportation Engineering in CSUST, and Ministry of Science Project with Montenegro (3-2).

\section{References}

[1] J. Y. Hao and Z. B. He, "A day-to-day invariant macroscopic fundamental diagrams for probe vehicles," IOP Conference Series: Earth and Environmental Science, vol. 189, no. 6, Article ID 062017, 2018. 
[2] Z. He, L. Yang, and W. Guan, "A day-to-day route choice model based on travellers' behavioural characteristics," Procedia-Social and Behavioral Sciences, vol. 138, pp. 738-747, 2014.

[3] W. Zhang, Z. He, W. Guan, and G. Qi, "Day-to-day rerouting modeling and analysis with absolute and relative bounded rationalities," Transportmetrica A: Transport Science, vol. 14, no. 3, pp. 256-273, 2018.

[4] Z. He, W. Guan, and S. Ma, "A traffic-condition-based route guidance strategy for a single destination road network," Transportation Research Part C: Emerging Technologies, vol. 32, pp. 89-102, 2013.

[5] D. F. Xie, X. M. Zhao, and Z. B. He, "Heterogeneous traffic mixing regular and connected vehicles: modeling and stabilization," IEEE Transactions on Intelligent Transportation Systems, vol. 20, no. 6, pp. 2060-2071, 2019.

[6] Z. B. He, W. Zhang, and N. Jia, "Estimating carbon dioxide emissions of freeway traffic: a spatiotemporal cell-based model," IEEE Transactions on Intelligent Transportation Systems, vol. 21, no. 5, pp. 1976-1986, 2020.

[7] Z. B. He, B. K. Chen, N. Jia, W. Guan, B. C. Lin, and B. H. Wang, "Route guidance strategies revisited: comparison and evaluation in an asymmetric two-route traffic network," International Journal of Modern Physics C, vol. 25, no. 4, Article ID 1450005, 2014.

[8] Z. He, S. Ma, and X. Tang, "Empirical study on the influence of learning ability to individual travel behavior," Journal of Transportation Systems Engineering and Information Technology, vol. 9, no. 2, pp. 75-80, 2009.

[9] F. Yang, An Evolutionary Game Theory Approach to the Dayto-Day Traffic Dynamics Dissertation, University of Wisconsin-Madison, Madison, WI, USA, 2005.

[10] F. Yang and D. Zhang, "Day-to-day stationary link flow pattern," Transportation Research Part B: Methodological, vol. 43, no. 1, pp. 119-126, 2009.

[11] X. Z. He and H. X. Liu, "Modeling the day-to-day traffic evolution process after an unexpected network disruption," Transportation Research Part B: Methodological, vol. 46, no. 1, pp. 50-71, 2012.

[12] J. Bie and H. K. Lo, "Stability and attraction domains of traffic equilibria in a day-to-day dynamical system formulation," Transportation Research Part B: Methodological, vol. 44, no. 1, pp. 90-107, 2010.

[13] R.-Y. Guo, H. Yang, and H.-J. Huang, "A discrete rational adjustment process of link flows in traffic networks," Transportation Research Part C: Emerging Technologies, vol. 34, pp. 121-137, 2013.

[14] Z. Huang, J. Wu, A. Kuang, S. Zhang, and Y. Xu, "Modeling and simulation of travelers' route choice behavior based on disequilibrium theory," Journal of System Simulation, vol. 30, no. 11, pp. 4067-4078, 2018.

[15] Z. Huang, J. Wu, R. Huang, and Y. Xu, "Network traffic flow evolution model based on disequilibrium theory," Mathematical Problems in Engineering, vol. 2018, Article ID 8478910, 10 pages, 2018.

[16] L. Wu, Z. Huang, and Y. Wang, "A dynamic evolution model of disequilibrium network traffic flow with quantity regulation of congestion," Journal of Traffic Transportation Engineering, vol. 18, no. 3, pp. 167-178, 2018.

[17] Z. Huang, D. Qin, and A. Kuang, "Capacity model of road network considering the impact of the autonomous vehicles," Journal of Changsha University of Science and Technology (Natural Science), vol. 15, no. 4, pp. 45-51, 2018.
[18] L. Wu, Z. Huang, J. Wu, Z. Gao, and D. Qin, "A day-to-day stochastic traffic flow assignment model based on mixed regulation," IEEE Access, vol. 8, pp. 12815-12823, 2020.

[19] L. Hu, X. Hu, J. Wan, M. Lin, and J. Huang, "The injury epidemiology of adult riders in vehicle-two-wheeler crashes in China, Ningbo, 2011-2015," Journal of Safety Research, vol. 72, pp. 21-28, 2020.

[20] L. Hu, J. Ou, J. Huang, Y. Chen, and D. Cao, "A review of research on traffic conflicts based on intelligent vehicles," IEEE Access, vol. 8, pp. 24471-24483, 2020.

[21] H. Zhao, Y. Li, W. Hao, S. Peeta, and Y. Wang, "Evaluating the effects of switching period of communication topologies and delays on electric connected vehicles stream with car-following theory," IEEE Transactions on Intelligent Transportation Systems, vol. 2020, no. 99, pp. 1-11, 2020.

[22] Y. Li, W. Chen, S. Peeta, and Y. Wang, "Platoon control of connected multi-vehicle systems under V2X communications: design and experiments," IEEE Transactions on Intelligent Transportation Systems, vol. 21, no. 5, pp. 1891-1902, 2020.

[23] H.-J. Huang and W. H. K. Lam, "Modeling and solving the dynamic user equilibrium route and departure time choice problem in network with queues," Transportation Research Part B: Methodological, vol. 36, no. 3, pp. 253-273, 2002.

[24] S. Peeta and T.-H. Yang, "Stability issues for dynamic traffic assignment," Automatica, vol. 39, no. 1, pp. 21-34, 2003.

[25] X. He, X. Guo, and H. X. Liu, "A link-based day-to-day traffic assignment model," Transportation Research Part B: Methodological, vol. 44, no. 4, pp. 597-608, 2010.

[26] W. Liu, X. Li, F. Zhang, and H. Yang, "Interactive travel choices and traffic forecast in a doubly dynamical system with user inertia and information provision," Transportation Research Part C: Emerging Technologies, vol. 85, pp. 711-731, 2017.

[27] D. Watling and M. L. Hazelton, "The dynamics and equilibria of day-to-day assignment models," Networks and Spatial Economics, vol. 3, no. 3, pp. 349-370, 2003.

[28] M. Ehrgott, J. Y. T. Wang, and D. P. Watling, "On multiobjective stochastic user equilibrium," Transportation Research Procedia, vol. 7, pp. 96-109, 2015.

[29] J. Long, J. Chen, W. Y. Szeto, and Q. Shi, "Link-based system optimum dynamic traffic assignment problems with environmental objectives," Transportation Research Part D: Transport and Environment, vol. 60, pp. 56-75, 2018.

[30] N. H. Hoang, H. L. Vu, and H. K. Lo, "An informed user equilibrium dynamic traffic assignment problem in a multiple origin-destination stochastic network," Transportation Research Part B: Methodological, vol. 115, pp. 207-230, 2018.

[31] L. Y. Xiang, G. Q. Guo, J. M. Yu, V. S. Sheng, and P. Yang, “A convolutional neural network-based linguistic steganalysis for synonym substitution steganography," Mathematical Biosciences and Engineering, vol. 17, no. 2, pp. 1041-1058, 2020.

[32] L. Xiang, X. Shen, J. Qin, and W. Hao, "Discrete multi-graph hashing for large-scale visual search," Neural Processing Letters, vol. 49, no. 3, pp. 1055-1069, 2019.

[33] C. X. Ma, W. Hao, F. Q. Pan et al., "Road screening and distribution route multi-objective robust optimization for hazardous materials based on neural network and genetic algorithm," PLoS One, vol. 13, no. 6, Article ID e0198931, 2018.

[34] M. S. Rahman, M. Abdel-Aty, J. Lee, and M. H. Rahman, "Safety benefits of arterials' crash risk under connected and automated vehicles," Transportation Research Part C: Emerging Technologies, vol. 100, pp. 354-371, 2019. 
[35] F. Chen, H. R. Peng, X. X. Ma, J. Y. Liang, W. Hao, and X. D. Pan, "Examining the safety of trucks under crosswind at bridge-tunnel section: a driving simulator study," Tunnelling and Underground Space Technology, vol. 92, Article ID 103034, 2019.

[36] W. Hao, C. Kamga, X. Yang et al., "Driver injury severity study for truck involved accidents at highway-rail grade crossings in the United States," Transportation Research Part F: Traffic Psychology and Behaviour, vol. 43, pp. 379-386, 2016.

[37] Q. Zeng, W. Gu, X. Zhang, H. Wen, J. Lee, and W. Hao, "Analyzing freeway crash severity using a Bayesian spatial generalized ordered logit model with conditional autoregressive priors," Accident Analysis \& Prevention, vol. 127, pp. 87-95, 2019.

[38] W. Hao, C. Kamga, and J. Daniel, "Driver's injury severity at U.S. highway-rail grade crossings by driver's age and gender," Journal of Safety Research, vol. 55, pp. 105-113, 2015.

[39] W. Hao and J. Daniel, "Driver's injury severity at U.S. highway-rail grade crossings by varied control devices," Journal of Safety Research, vol. 51, pp. 41-48, 2014.

[40] Z. Wang, J. Yu, W. Hao et al., "Two-step coordinated optimization model of mixed demand responsive feeder transit," Journal of Transportation Engineering, Part A: Systems, vol. 146, no. 3, Article ID 04019082, 2020.

[41] J. J. Tang, Y. W. Wang, W. Hao, F. Liu, H. L. Huang, and Y. H. Wang, "A mixed path size logit-based taxi customersearch model considering spatio-temporal factors in route choice," IEEE Transactions on Intelligent Transportation Systems, vol. 21, no. 4, pp. 1347-1358, 2020.

[42] W. Hao, Y. J. Lin, Y. Cheng, and X. F. Yang, "Signal progression model for long arterial: intersection grouping and coordination," IEEE Access, vol. 6, pp. 30128-30136, 2018.

[43] H.-J. Huang and Z.-C. Li, "Discrete optimization: a multiclass, multicriteria logit-based traffic equilibrium assignment model under ATIS," European Journal of Operational Research, vol. 176, no. 3, pp. 1464-1477, 2007.

[44] X. Zhang, H. Yang, and H.-J. Huang, "Multiclass multicriteria mixed equilibrium on networks and uniform link tolls for system optimum," European Journal of Operational Research, vol. 189 , no. 1, pp. 146-158, 2008.

[45] L. Han, H. Sun, J. Wu, and C. Zhu, "Day-to-day evolution of the traffic network with advanced traveler information system," Chaos, Solitons \& Fractals, vol. 44, no. 10, pp. 914-919, 2011.

[46] B. Zhou, M. Xu, Q. Meng, and Z. Huang, "A day-to-day route flow evolution process towards the mixed equilibria," Transportation Research Part C: Emerging Technologies, vol. 82, pp. 210-228, 2017.

[47] M. Xu, Q. Meng, and Z. Huang, "Global convergence of the trial-and-error method for the traffic-restraint congestionpricing scheme with day-to-day flow dynamics," Transportation Research Part C: Emerging Technologies, vol. 69, pp. 276-290, 2016.

[48] S. A. Bagloee, M. Sarvi, M. Patriksson, and A. Rajabifard, "A mixed user-equilibrium and system-optimal traffic flow for connected vehicles stated as a complementarity problem," Computer-Aided Civil and Infrastructure Engineering, vol. 32, no. 7, pp. 562-580, 2017.

[49] P. Delle Site, "A mixed-behaviour equilibrium model under predictive and static advanced traveller information systems (ATIS) and state-dependent route choice," Transportation Research Part C: Emerging Technologies, vol. 86, pp. 549-562, 2018.
[50] Z. X. Huang, X. J. Jiang, and J. H. Wu, "An evolution model for network traffic flow based on price-quantity regulation," Journal of Management Sciences in China, vol. 20, no. 8, pp. 102-111, 2017.

[51] X. J. Jiang, Z. X. Huang, and Z. Y. Zhao, "A traffic flow evolution process toward mixed equilibrium with multicriteria of route choice behaviour," Journal of Advanced Transportation, vol. 2020, Article ID 1720949, 17 pages, 2020. 